Article

\title{
On the Interaction of Side-By-Side Circular Cylinders in Viscoplastic Fluids
}

\author{
Naser Hamedi *(D) and Lars-Göran Westerberg \\ Department of Engineering Sciences and Mathematics, Division of Fluid and Experimental Mechanics, \\ Luleå University of Technology, SE-971 87 Luleå, Sweden; Lars-Goran.Westerberg@ltu.se \\ * Correspondence: naser.hamedi@ltu.se
}

Received: 12 April 2019; Accepted: 17 May 2019; Published: 21 May 2019

check for updates

\begin{abstract}
In this paper, the static interaction of a train of three cylinders in a Bingham fluid is studied numerically using Computational Fluid Dynamics. The variation of drag forces for the cylinders in several configurations is investigated. Positions of the particles in relation to the reference particle are recognized by the separation distance between the cylinders. A steady state field is considered, with Bingham numbers between 5 and 150. Several separation distances $(d)$ were considered, such that $2.0 D \leq d \leq 6.0 D$ where $D$ is the cylinder diameter. The Reynolds number was chosen in the range of $5 \leq R e \leq 40$. In particular, the effect of the separation distance, Reynolds number and Bingham number on the shape and size of the unyielded regions was investigated. The functional dependence of this region and the drag coefficient is explored. The present results reveal the significant influence of the gap between the cylinders on the drag force and the shape of the unyielded regions surrounding the cylinders. It was found that there are several configurations in which the drag forces over the first and the third cylinders are almost equal depending on variation of the $B i, R e$ and the separation distance.
\end{abstract}

Keywords: particle interaction; viscoplastic fluid; Bingham fluid; computational fluid dynamics

\section{Introduction}

The flow around bluff bodies have been a matter of interest for researchers for many years. The study of non-Newtonian fluids past particulate objects are rapidly increasing. Such flow scenarios can be found in nature, and not the least in many different engineering applications. In nature, the flow of crude oil passed rocks is one example. In the industry, a few applications are flow in porous media such as during composite material manufacturing, flow of food materials through shells, flow in tube heat exchanger, and lubricant flow in machine elements. In the rheological behavior of materials like concrete, tomato paste and many dairy products, a so-called yield stress at low shear rates is often observed. This phenomenon has been studied by a number of researchers; see Refs. [1-5] for some central contributions, and there is an ongoing discussion whether a true yield stress exists. Regarding the yield stress, two main hypotheses have been proposed "true" yield stress and "apparent" yield stress. The first hypothesis anticipates that under a certain threshold shear stress value, the fluid exhibits a solid-like behavior while exceeding this threshold, the fluid starts to flow. According to the second hypothesis, all solid and fluid materials can creep and ultimately flow, provided the time scale is long enough [6,7]. In other words, for the case of a vanishing shear rate, the fluid can still flow and with increased shear rate, the apparent viscosity quickly and asymptotically decreases. The Bingham [8] and Herschel-Bulkley [9] rheology models are both based on hypothesis one, i.e., a true yield stress. 
In the Bingham model, the shear stress $\tau$ is assumed to be linearly proportional to the shear rate $\dot{\gamma}$, after it exceeds a certain Bingham yield stress $\left(\tau_{0}\right)$ such that

$$
\begin{cases}\dot{\gamma}=0 ; & \tau \leq \tau_{0} \\ \tau=\tau_{0}+\mu \dot{\gamma} ; & \tau>\tau_{0}\end{cases}
$$

where $\mu$ is the Newtonian viscosity. In order to consider the non-linear behavior of the fluid in the yielded part, the Herschel-Bulkley model is used according to:

$$
\begin{cases}\dot{\gamma}=0 ; & \tau \leq \tau_{0} \\ \tau=\tau_{0}+K \dot{\gamma}^{n} ; & \tau>\tau_{0}\end{cases}
$$

Here $K$ is the consistency index and $n$ the Power-law index. It should be noted that in both the Bingham and Herschel-Bulkley models, the yield stress is defined by the von Mises criteria which states that the yielding of a material begins when the second deviatoric stress invariant tensor reaches a critical value. According to Equations (1) and (2), the Bingham and Herschel-Bulkley rheology models are discontinuous, as there is no transition from unyielded to yielded built into the models. Papanastasiou [10] proposed a modified Bingham model in which the viscosity function smoothly changes for the whole domain such that:

$$
\tau=\left(\mu+\tau_{0} \frac{1-\exp (-m \dot{\gamma})}{\dot{\gamma}}\right) \dot{\gamma}
$$

where $m$ is a sufficiently large number to guarantee large apparent viscosity $(\eta)$ at vanishing strain rate. This model is an example of a so-called regularize model, which is the most widely used approach to avoid the discontinuity. Some related works in this area are found in Refs. [11-14].

There are numerous applications in nature and industries such as particulate foods in non-Newtonian liquids or crude oil flow with rocks. The first work on the study of the flow past a Newtonian fluid is reported by Stokes (1851), where Stokes' law [15] was presented to calculate the drag force on a spherical object. In fact, the bulk of the studies in this research field is concerned with the Newtonian flow past spheres; see, e.g., [16-19]. The first study of creeping flow of a Bingham fluid past an object is by Beris et al. [20], who presented a Finite Element (FEM)/Newtonian method to solve the flow field including the yield surfaces in the creeping flow over a sphere. They found a non-dimension value for the critical yield stress value for which below this value the material acts as a solid in the domain. In addition, a detailed flow pattern around the sphere as well as the results for the drag coefficient was presented.

The earliest result for the solution of viscoplastic fluids around a 2D cylinder has been reported by Adachi and Yoshioka [21]. Using the slip-line analysis and the minimum- and maximum stress principles, they calculated upper and lower bounds for the drag coefficient and compared these values with each other. Moreover, they calculated the fluid region and the rigid region around the cylinder-i.e., the corresponding yield surface. In another work by Mitsoulis [22], the creeping flow of a Bingham fluid past a circular cylinder and the wall effects were investigated by FEM simulations. The blockage ratio varied between 2-50 in their study. Based on numerical prediction, they determined the drag coefficient as a function of the Bingham number for different gap/cylinder ratios. In addition, they examined un-sheared regions around the cylinder for various Bingham numbers. Different studied have been performed for the flow of Newtonian fluids past dual cylinders. Sheldon and Green [23] studied numerical simulation of the Newtonian flow around two-dimensional tandem circular cylinders perpendicular to the flow direction for the case of $1 \leq R e \leq 20$ and surface-to-surface separation distance to cylinder diameter ratio between 0.1 and 30 . They investigated the effect of the gap distance between the cylinders on the lift and drag coefficient. Significant change of the lift and drag coefficients was found for the range of the Reynolds numbers studies. Due to the reduced viscous 
forces at small gap distances, they found a remarkable reduced drag force comparing to the single cylinder. For the lift coefficient variation with the gap distance, they found a sigmoidal pattern.

On the topic of viscoplastic fluid flow past dual cylinders, Jossic and Magnin [24] studied the interaction of two parallel cylinders, both numerically and experimentally, where $1 \leq B i \leq 40$ and compared the results with a singular cylinder. Using the correlation between the drag coefficient and the Bingham number, they estimated a distance where there is no interaction between the two cylinders. They also investigated the influence of the slip condition on the drag forces. In the recent research, Koblitz et al. [25] presented the results of DNS simulations of interacting circular cylinders in three different cases whereas a Bingham fluid was changed between 0-2000 and the separation distance was kept constant at $1 \%$ of the cylinder radius in which the flow type was so called squeeze flow. They showed that the asymptotic lubrication solution is used in presence of the macroscopic flow assumption.

In this paper, we describe numerical simulations of the viscoplastic flow around three two-dimensional side-by-side circular cylinders with gap spacing $3.0 D \leq d \leq 5.0 D, D$ being the cylinder diameter, and a Bingham number $10 \leq B i \leq 150$. The Reynolds number is $5 \leq R e \leq 40$. In Section 2, we describe the numerical method and computational domain used for the simulations. Sections 4 and 5 discusses the results and Section 6 is comprised of a summary and conclusions. Although there are some studies made on the experimental or numerical approach of Newtonian fluid past three stationary spheres in steady or turbulent regions [26,27], to the best of the authors' knowledge, no study has been performed on the investigation of the viscoplastic fluid over three or more fixed stationary objects so far. Three cylinder objects enable us to further investigate the interaction between the objects and compare the outcomes with the single cylinder ones. It is important and of particular interest to build an understanding of how a train of cylinders affects the flow. The evolution and distribution of the yielded and unyielded regions are important for an understanding of the flow evolution in such domains.

\section{Governing Equations and Numerical Models}

The description below is based on the assumptions that the flow is steady, laminar and without secondary motions, which is indeed the case considering the very low Reynolds number for the flow in the domain. Non-dimensionalising the governing equations using the diameter of the cylinder $D$ as characteristic length scale and the inlet velocity $u$ as characteristic velocity we get:

$$
\begin{gathered}
\nabla \cdot \mathbf{u}=0 \\
(\mathbf{u} \cdot \nabla) \mathbf{u}=-\nabla p+\frac{1}{\operatorname{Re}} \nabla \cdot \tau
\end{gathered}
$$

As previously mentioned, in this work, the shear thinning fluid is modelled using a so-called regularized Bingham Papanastasiou viscosity model expression [10]. The parameter $m$ is considered sufficiently large to guarantee large apparent viscosity $(\eta)$ at vanishing strain rate. Figure 1 shows the shear stress curve versus shear rate for different values of $m$. For the current study, $m=1000$ was considered in all simulations, which does not show significant results compared to the ideal Bingham model.

The shear rate $(\dot{\gamma})$, is calculated using the second invariant of the rate of strain tensor $S_{i j}$ [28]:

$$
\begin{aligned}
& I_{2}=S_{i j} S_{i j} \\
& \dot{\gamma}=\sqrt{\frac{I_{2}}{2}}
\end{aligned}
$$


To distinguish the yielded region from the non-yielded one considering the yield stress definition, one can use the criterion in Equation 8. The material consequently flows when the magnitude of the stress tensor exceeds the yield stress value in accordance with:

$$
\begin{cases}\text { Non-yielded; } & \tau \leq \tau_{0} \\ \text { yielded; } & \tau>\tau_{0}\end{cases}
$$

In this paper, the OpenFOAM open source Computational Fluid Dynamics (CFD) software [29] was employed to model the viscoplastic fluid flow past the three tandem cylinders. The finite volume solver, which uses a non-staggered grid, calculates the mass and momentum equations in the discretized form, which guarantees the conservation of fluxes through the control volume. Diffusion and pressure gradient terms were evaluated by a second order interpolation. The PISO scheme was employed for the pressure-velocity coupling.

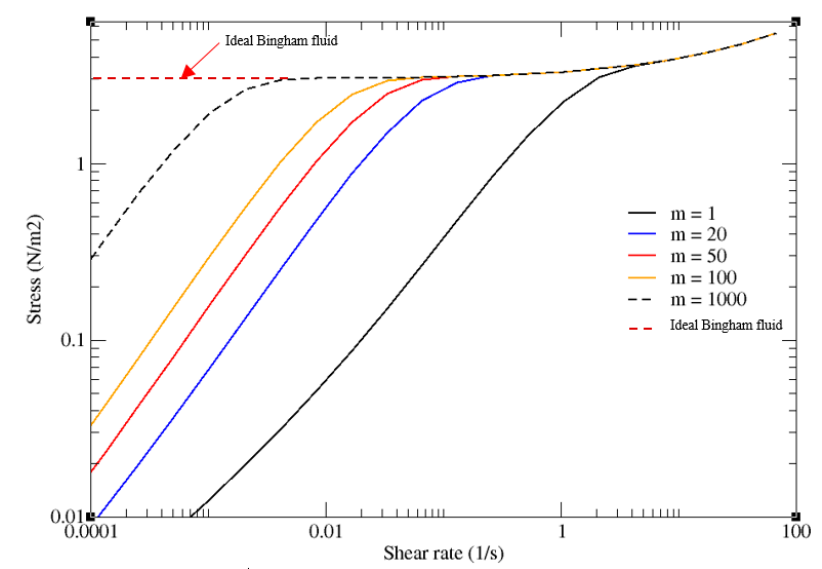

Figure 1. Shear stress versus shear rate for different values of $m$ according to the Papanastasiou Bingham model.

\section{Problem Setup}

Three equally sized cylinders with diameter $D$ are taken into account at several relative positions in a rectangular domain and Cartesian coordinates. Figure 2 shows the domain and cylinders positions. The positions are solely defined using separation distance $d$. Simulations are carried out for $d=2.0 D$, $3.0 D, 4.0 D, 5.0 D$ and $6.0 D$, respectively. The dimensions of the $2 \mathrm{D}$ computational domain are $15 \times 40$. The results of the grid independency of the computational domain are shown in Figure 2. The grid resolution of $h=D / 20$ is used for the computation; see Figure 3.

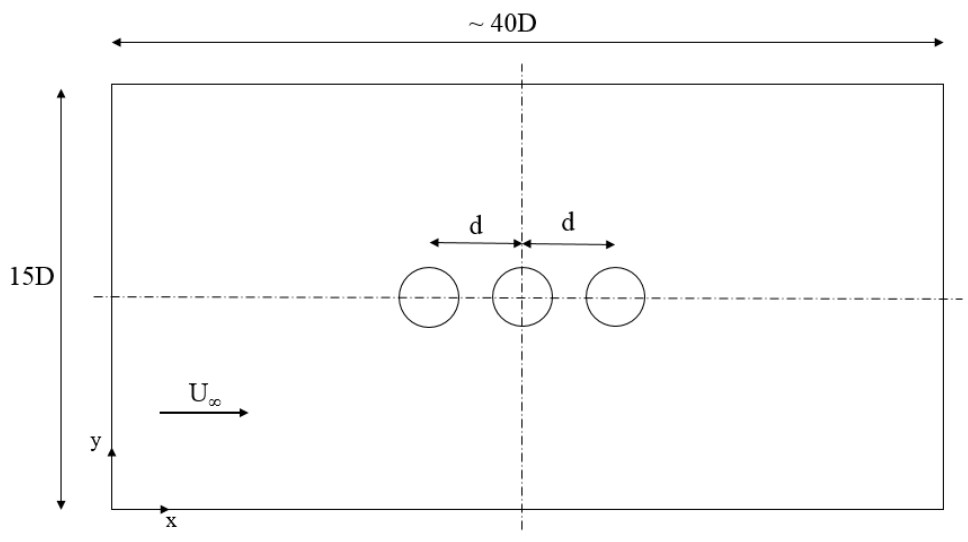

Figure 2. Problem setup and arrangement of the three cylinders. 
A uniform velocity profile is applied at the inlet, a Dirichlet condition at the outlet, slip boundary conditions at the lateral boundaries, and a no-slip boundary condition on the cylinders surface.

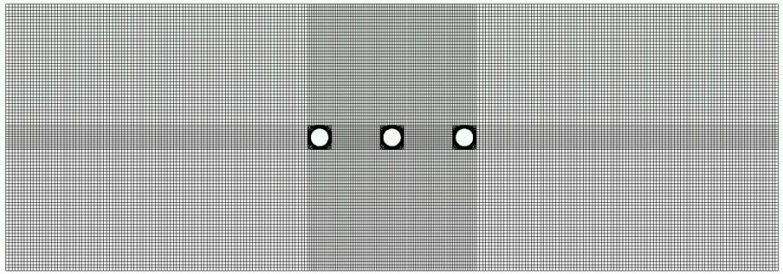

(a)

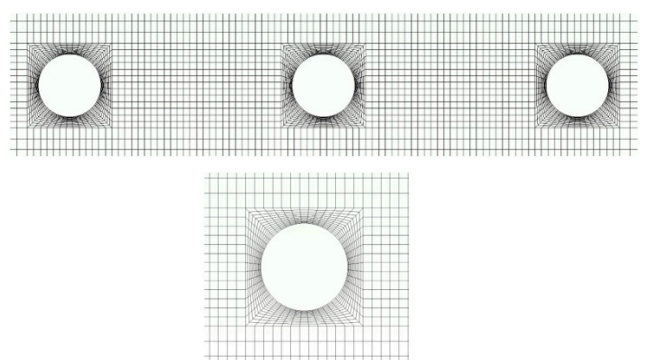

(b)

Figure 3. (a) Discretized model for the three cylinders with $d=4.0 D,(\mathbf{b})$ a closer view of the grids for single cylinder and triple cylinders.

A grid sensitivity study of all computational domains used in this study was obtained. Various simulations of the Newtonian flow past the single cylinder and triple cylinders was performed and the drag coefficient was selected as a criterion to find a grid independent computational domain. It was found that there were only minor differences in the drag coefficient for the three finest resolution. As previously mentioned, the grid resolution of $D / 20$ was used for all computational domains.

\section{Results}

\subsection{Single Cylinder}

For validating the non-Newtonian solver, the drag coefficient results of the single cylinder in viscoplastic media were compared with the results of the former researchers. Table 1 shows the results for viscoplastic fluid flow (Bingham number 10) for three Reynolds numbers- $R e=5,20$ and 40 . The results agree well with previous studies.

Table 1. Drag variation for three Reynolds number at Bingham number 10 in the current study compared to the previous works.

\begin{tabular}{cccc}
\hline Reference & $\boldsymbol{R} \boldsymbol{e}=\mathbf{1 0}$ & $\boldsymbol{R} \boldsymbol{e}=\mathbf{2 0}$ & $\boldsymbol{R} \boldsymbol{e}=\mathbf{4 0}$ \\
\hline Present work & 33.30 & 17.11 & 8.85 \\
Mossaz et al. [30] & 34.79 & 17.21 & 9.43 \\
Nirmalkar and Chhabra [31] & 33.11 & 17.00 & 8.97 \\
Takur et al. [32] & 34.83 & 17.20 & 9.37 \\
\hline
\end{tabular}

\subsubsection{Drag Variation}

Figure 4 shows the drag variation over a single cylinder where the Bingham number and Reynolds number are varied such that $5 \leq R e \leq 40$ and $5 \leq B i \leq 150$. According to Figure 4 -and as expected-the drag coefficient is increased with an increased Bingham number. However, the variation of the drag coefficient is more pronounced for the lower Reynolds numbers. In other words, for lower Reynolds numbers, the effect of the Bingham number is dominating compared to the range of higher Reynolds numbers. 


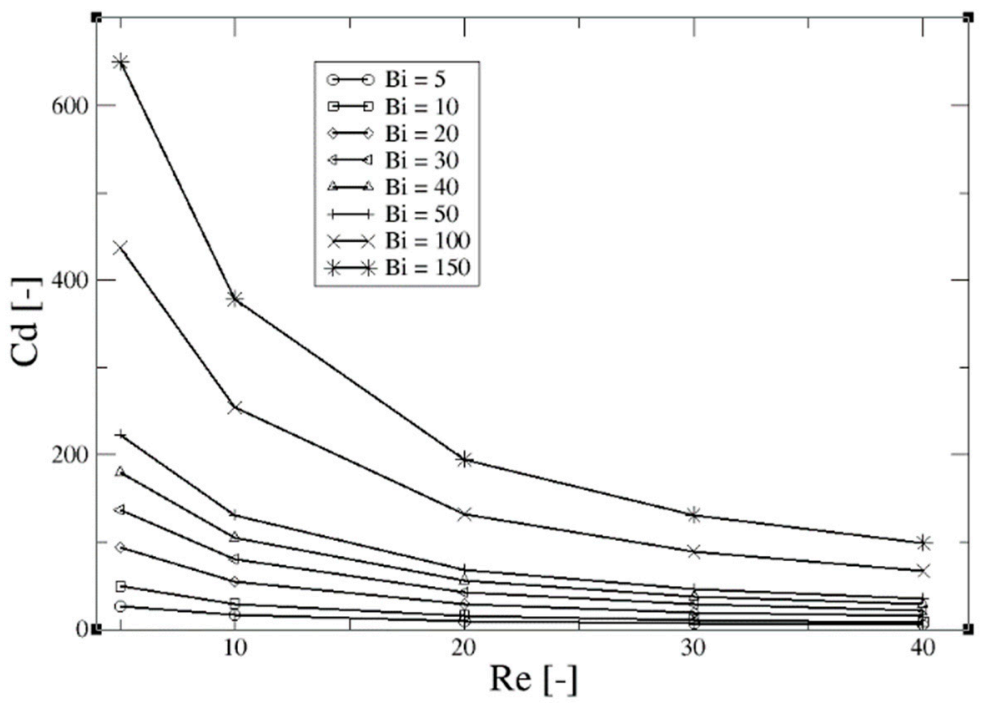

Figure 4. Variation of the drag coefficient for a single cylinder where the Reynolds number $(R e)$ and Bingham number $(B i)$ are varied.

\subsubsection{Non-Yielded Region Variation}

Figure 5 shows the unyielded region (shaded) observed in the viscoplastic fluid past the single sphere. The unyielded region appeared around the cylinder is varied by changing the Reynolds and the Bingham numbers. Island and polar caps regions can be observed as well. Although increasing the Bingham number, increases the unyielded regions around the cylinder, the size and the shape of these regions are different and does not follow a similar pattern in different Reynolds numbers. When $R e>10$, two small unyielded regions appear at top and bottom of the cylinder, which were not seen in the lower Reynolds number. In addition, while the Bingham number increases, the unyielded region gets smaller at flow direction, while at the vertical direction, it remains almost constant.

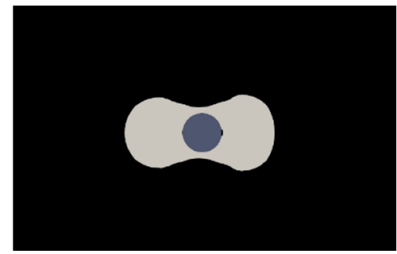

$\operatorname{Re}=5, B i=5$

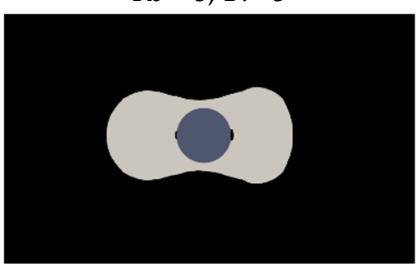

$\operatorname{Re}=10, B i=5$

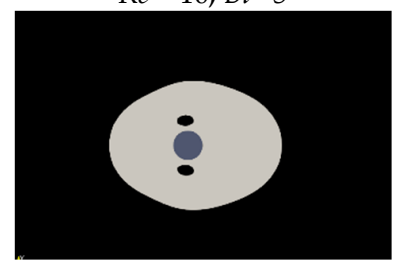

$\operatorname{Re}=20, B i=5$

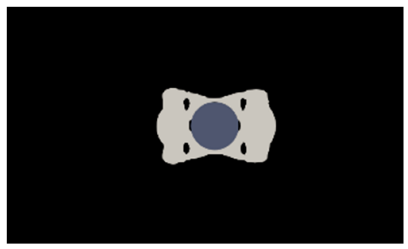

$\operatorname{Re}=5, B i=50$

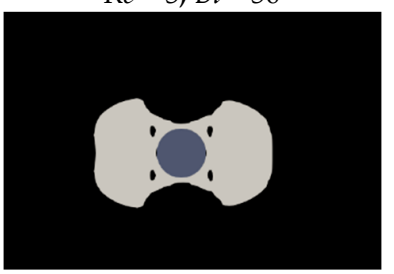

$R e=10, B i=50$

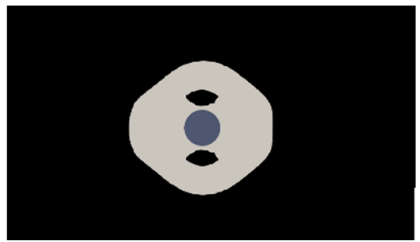

$\operatorname{Re}=20, B i=50$

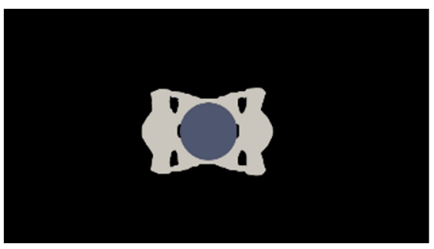

$\operatorname{Re}=5, B i=100$

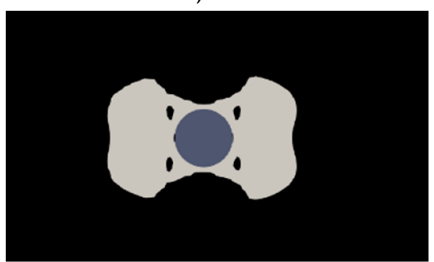

$\operatorname{Re}=10, B i=100$

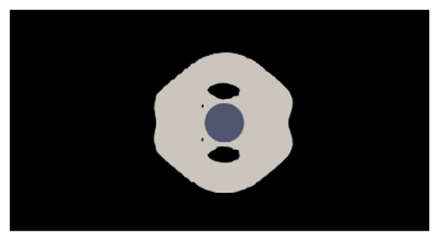

$\operatorname{Re}=20, B i=100$

Figure 5. Cont. 


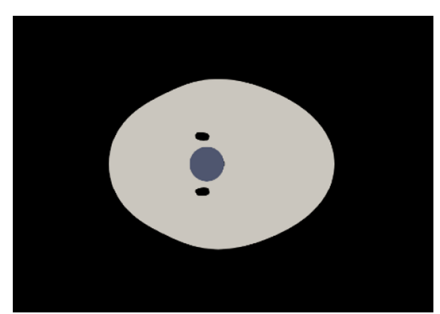

$R e=30, B i=5$

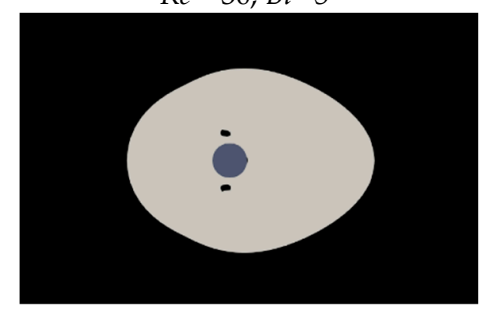

$\operatorname{Re}=40, B i=5$

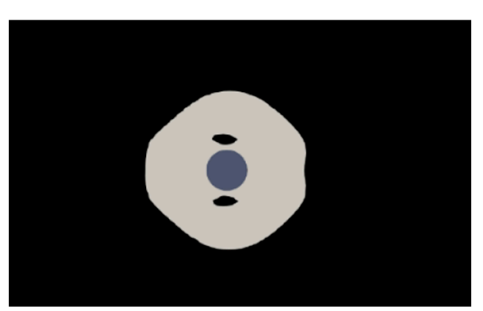

$\operatorname{Re}=30, B i=50$

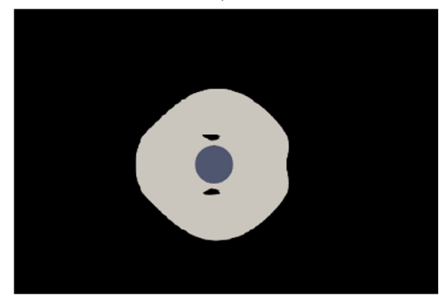

$\operatorname{Re}=40, B i=50$

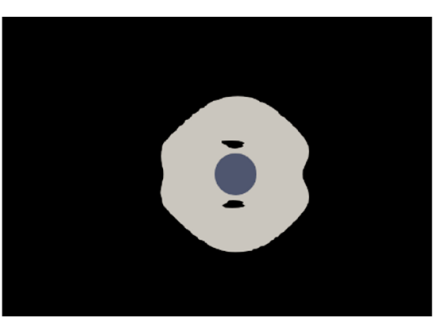

$R e=30, B i=100$

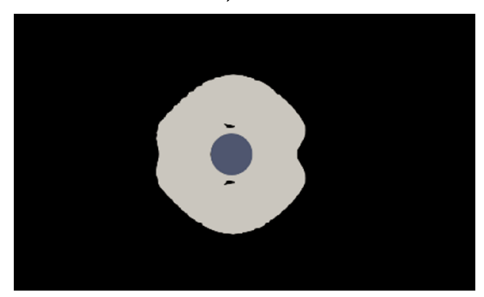

$\operatorname{Re}=40, B i=100$

Figure 5. Simulation results to show the progressive growth of the unyielded region (shaded).

It should be noted that the small zig-zag lines divide the unyielded and yielded regions, relating to the use of higher values of $m$ coefficient $(m=1000 \mathrm{~s}$ ) in Equation (3), which generate non-smooth lines in the solution. These lines has been observed in previous research [33].

\subsection{Three Cylinders}

\subsubsection{Drag Coefficients}

Figure 6 shows the variation of the drag coefficient for the three cylinders, where the separation distances are changed to $2.0 D \leq d \leq 6.0 D$ and $5 \leq B i \leq 100$. Like the drag alteration for the single cylinder, the drag coefficient is increased as the Bingham number increased. The cylinder in the middle position is also significantly affected by these parameters. However, the effect is varied by the variation of both Reynolds number and the Bingham number. This will be discussed later in this study.
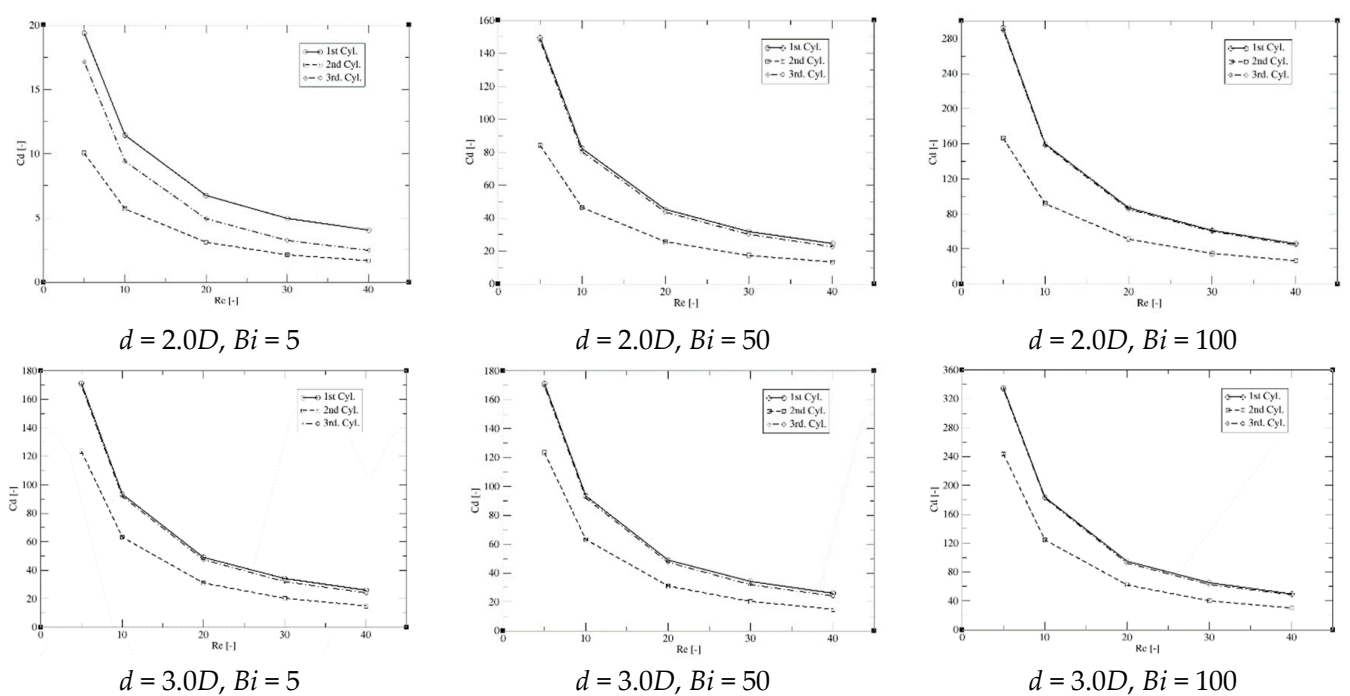

Figure 6. Cont. 

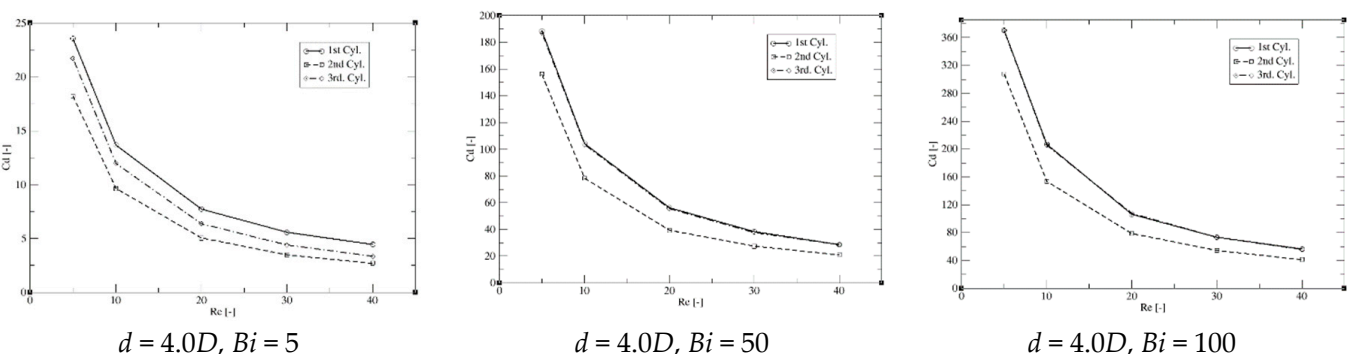

$d=4.0 D, B i=5$

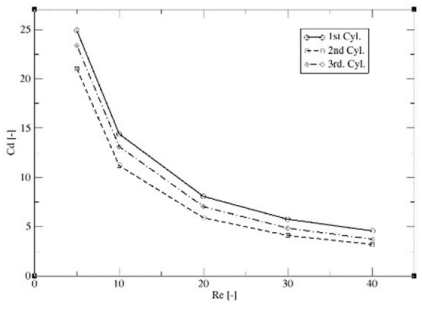

$d=4.0 D, B i=50$

$d=4.0 D, B i=100$
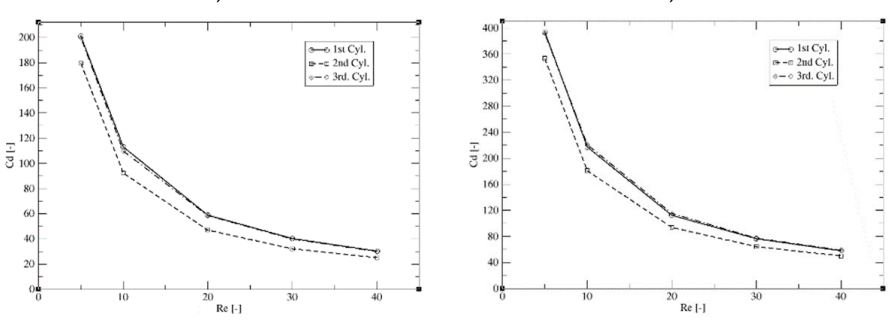

$d=5.0 D, B i=5$

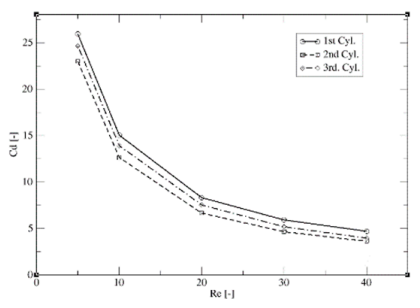

$d=5.0 D, B i=50$

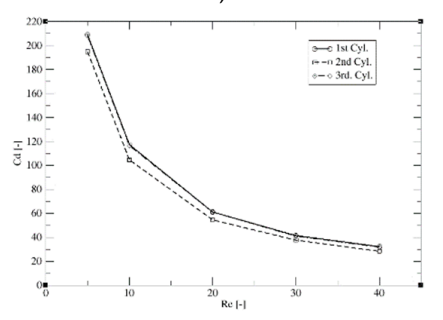

$d=5.0 D, B i=100$

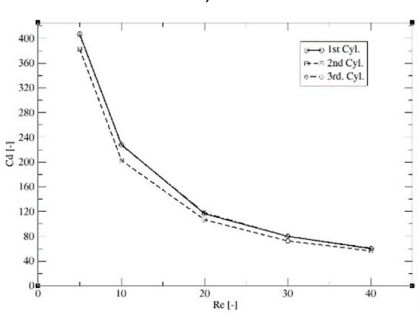

$d=6.0 D, B i=50$

$d=6.0 D, B i=100$

Figure 6. Drag variation over three cylinders for various separation distances and Bingham numbers.

\subsubsection{Unyielded Region}

Figures 7-10 show the growth of the unyielded region around three cylinders in tandem position for separation distances $d=2.0 D, 3.0 D, 4.0 D$ and $6.0 D, B i=5,50$ and 100 and $R e=5,20$ and 40 . As shown in the figures, increasing the Bingham number or decreasing the Reynolds number, both enhances the yield regions around and between the cylinders.

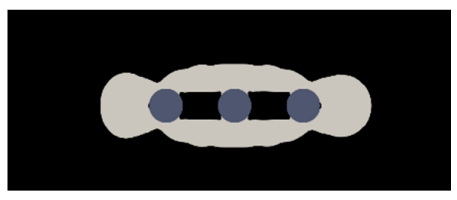

$\operatorname{Re}=5, B i=5$

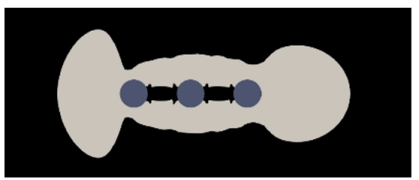

$R e=20, B i=5$

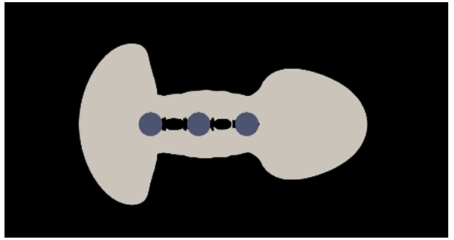

$R e=40, B i=5$

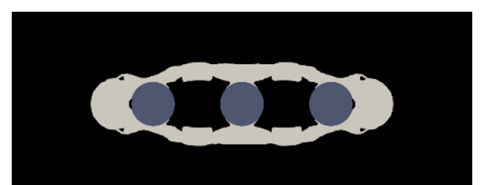

$\operatorname{Re}=5, B i=50$

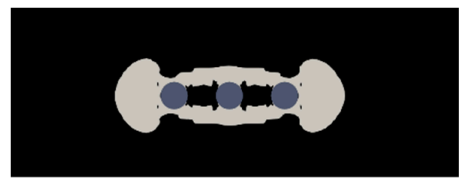

$R e=20, B i=50$

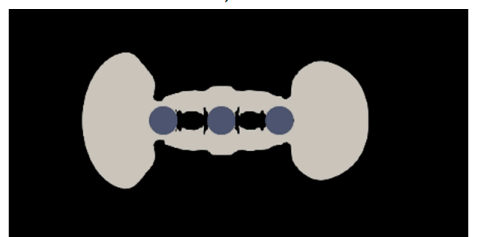

$R e=40, B i=50$

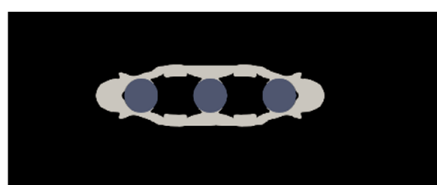

$\operatorname{Re}=5, B i=100$

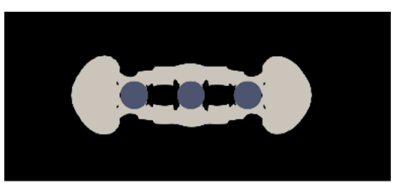

$R e=20, B i=100$

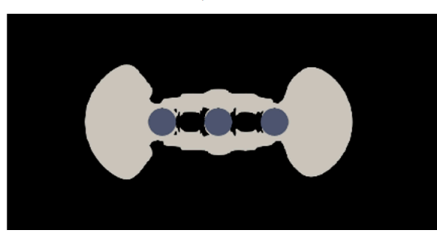

$R e=40, B i=100$

Figure 7. Progressive growth of the unyielded region (shaded) with the increasing Bingham number and decreasing Reynolds number where separation distance $d=2.0 \mathrm{D}$. 


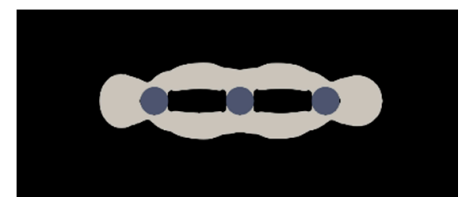

$R e=5, B i=5$

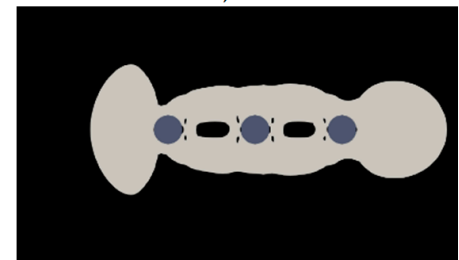

$R e=20, B i=5$

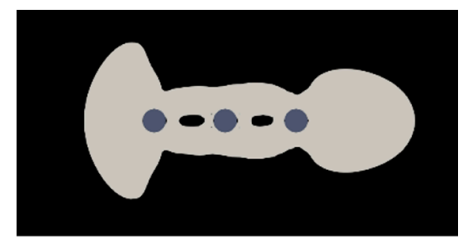

$R e=40, B i=5$

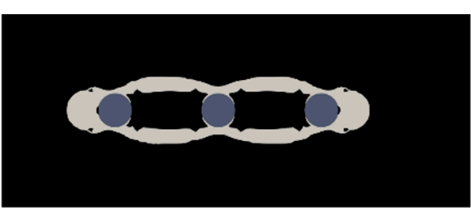

$R e=5, B i=50$

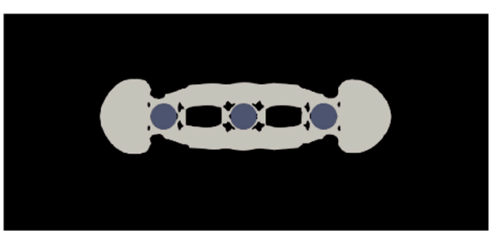

$R e=20, B i=50$

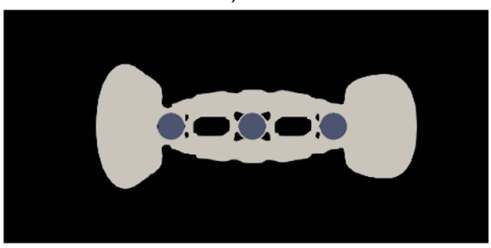

$R e=40, B i=50$

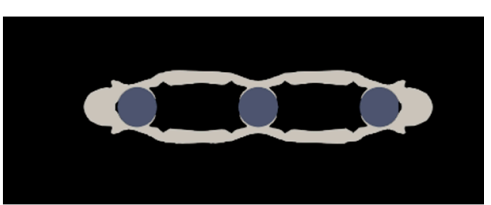

$R e=5, B i=100$

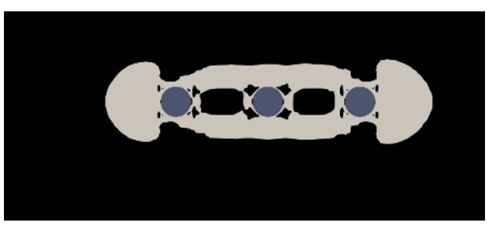

$R e=20, B i=100$

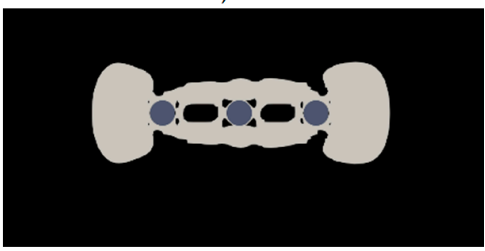

$R e=40, B i=100$

Figure 8. Progressive growth of the unyielded region (shaded) with the increasing Bingham number and decreasing Reynolds number where separation distance $d=3.0 D$.

From the figures, one can notice the separation distance influences on the breakage of the unyielded region between the cylinders. This phenomenon is observed for separation distance greater than 3.0D. For example, for $d=4.0 D$, the continuous unyielded region, which was previously observed for all cases in $d=2.0 D$ and $d=3.0 D$, were broken and depending on the Bingham number and the Reynolds number, these region sizes are varied. The size of the unyielded region between the cylinders are more noticeable for cases with the lowest Reynolds number and the highest Bingham number where $d \geq 4.0 D$. In these cases, the size of the un-sheared zone is significantly larger than the other cases (e.g., see cases with $R e=5, B i=150$ in Figures 9 and 10).

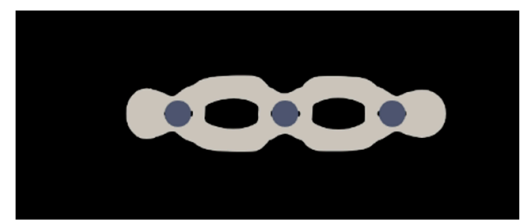

$R e=5, B i=5$

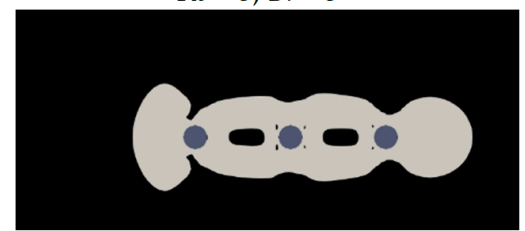

$R e=20, B i=5$

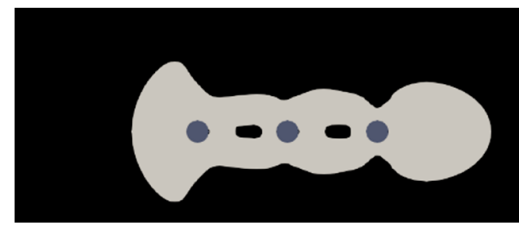

$R e=40, B i=5$

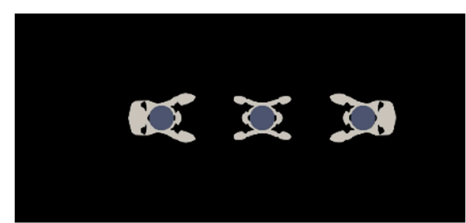

$R e=5, B i=50$

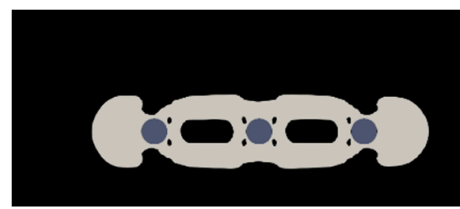

$R e=20, B i=50$

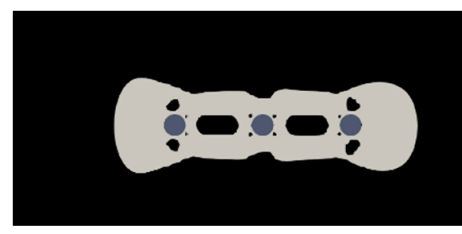

$R e=40, B i=50$

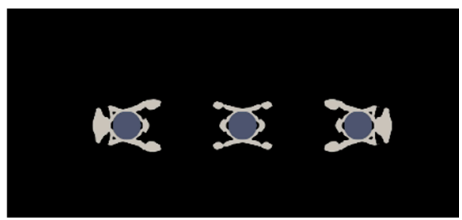

$R e=5, B i=100$

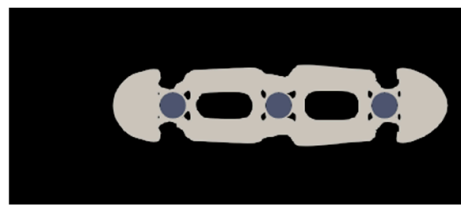

$R e=20, B i=100$

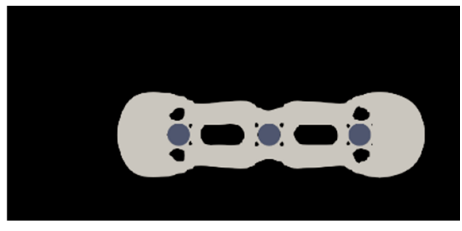

$R e=40, B i=100$

Figure 9. Progressive growth of the unyielded region (shaded) with the increasing Bingham number and decreasing Reynolds number where $d=4.0 D$. 


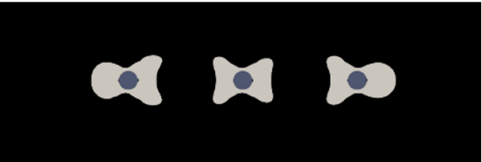

$R e=5, B i=5$

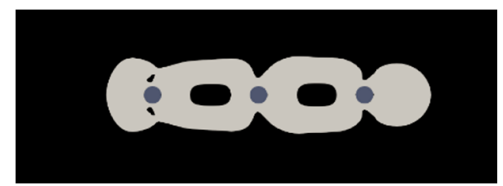

$R e=20, B i=5$

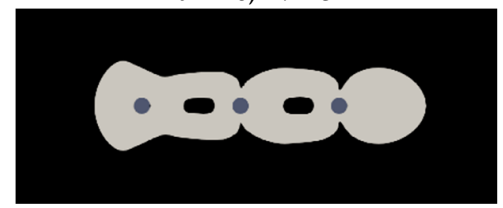

$R e=40, B i=5$
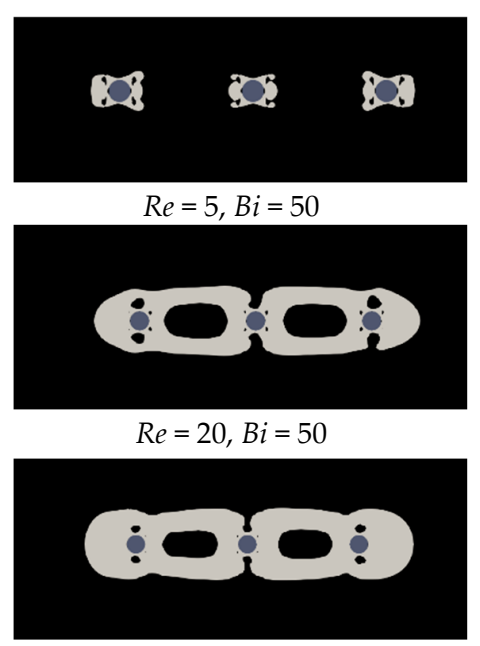

$R e=40, B i=50$

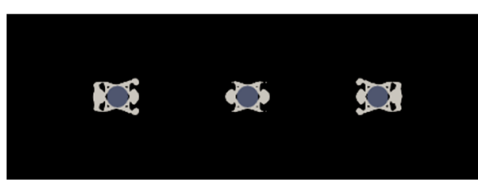

$R e=5, B i=100$

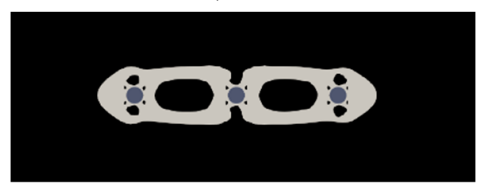

$\operatorname{Re}=20, B i=100$

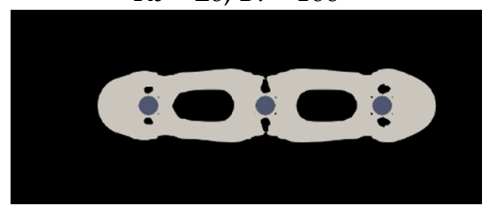

$\operatorname{Re}=40, B i=100$

Figure 10. Progressive growth of the unyielded region (shaded) with the increasing Bingham number and decreasing Reynolds number where $d=6.0 \mathrm{D}$.

\subsubsection{Velocity Distribution}

Figure 11 shows the velocity distribution of the three cylinders in separation distance $d=4.0$, three Reynolds number $R e=5,20$ and 40, and three Bingham number $B i=5, B i=50$ and $B i=100$. As shown in the plots, the distribution of the velocity is different in various Reynolds numbers and Bingham numbers. As is shown in the figure, by increasing the Bingham number, the fluid shows a more symmetrical shape, which is related to the developing unyielded areas and the plug flow around the cylinders.

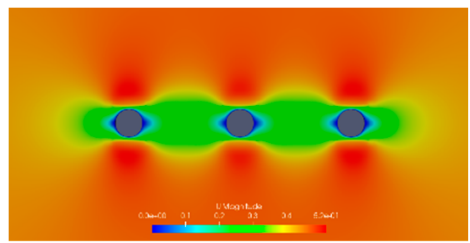

$\operatorname{Re}=5, B i=5$

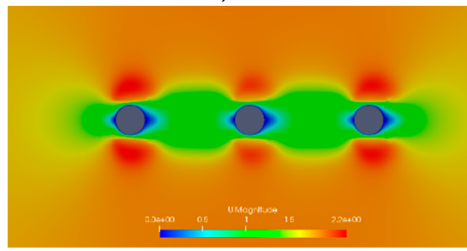

$R e=20, B i=5$

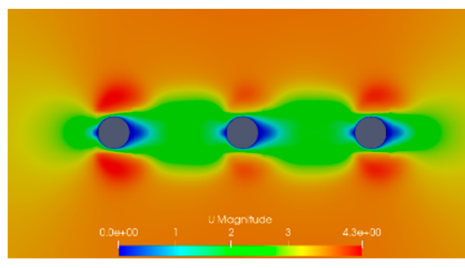

$\operatorname{Re}=40, B i=5$

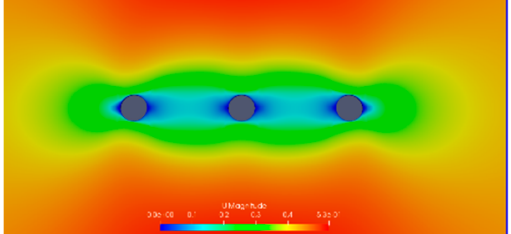

$\operatorname{Re}=5, B i=50$

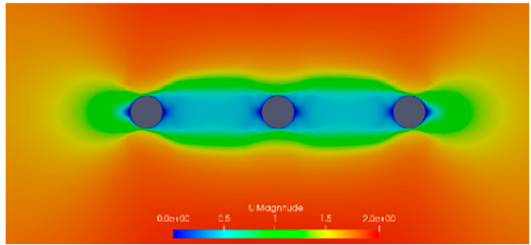

$R e=20, B i=50$

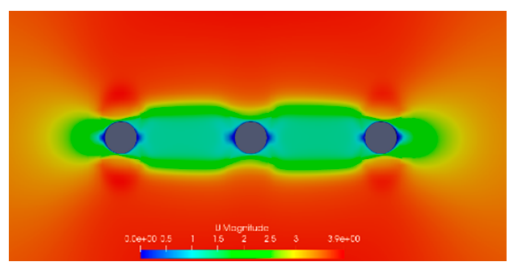

$\operatorname{Re}=40, B i=50$

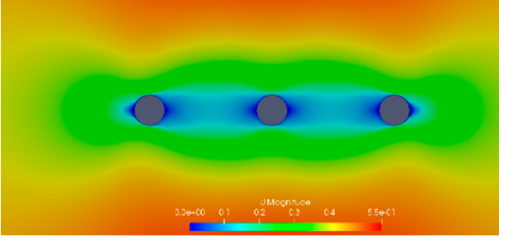

$\operatorname{Re}=5, B i=100$

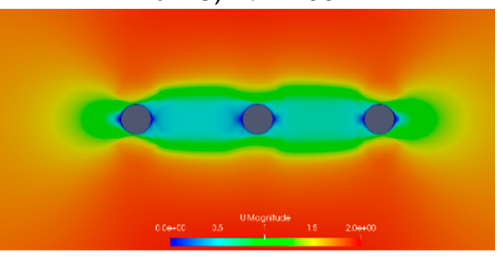

$\operatorname{Re}=20, B i=100$

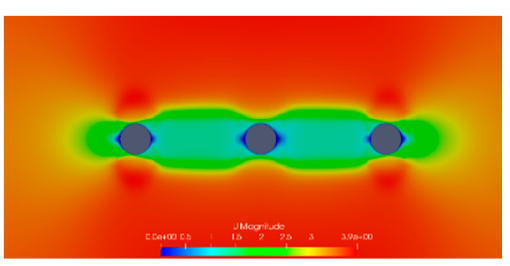

$\operatorname{Re}=40, B i=100$

Figure 11. Velocity profile around three cylinder in three Bingham numbers ( $\mathrm{Bi}=5,50$ and 100) and three Reynolds number $(\operatorname{Re}=5,20$ and 40) where $d=4.0 D$. 


\section{Discussion}

The results in the previous section comprise of the outcome of viscoplastic fluid flow past singleand triple cylinders, respectively. The drag coefficient of the single cylinder was compared with previous results and the results were found to agree well. Concerning the drag coefficient for various Bingham numbers, it was shown that its variation is more pronounced for lower Reynolds numbers, which is due to the dominant viscous forces in the actual range of Reynolds numbers. Likewise, the unyielded region was reported for various Reynolds and Bingham numbers. Island and polar caps regions, which were reported in previous research [12,21,33,34], were observed in this study as well. The structure of the unyielded regions are very similar to the earlier findings cited above.

Regarding the viscoplastic fluid flow past triple cylinders (as mentioned in the Introduction), to the best of the authors' knowledge, this is the first study that examines the interaction between the cylinders and the unyielded regions around the objects in the presence of the viscoplastic fluid. The drag coefficient of three cylinders was depicted in various configurations. The higher the Reynolds number, the more difference between the drag coefficients of the cylinder in the middle position and the first/third position was observed. It should be noted that this pattern is repeated for all separation distances, but the rate of this difference is a matter of importance. In other words, for the smaller separation distances, this influence is more than the same effect for the bigger separation distances. It is worthwhile to note that due to the effect of the viscoplastic properties of the carrying fluid, by decreasing the Bingham number, the difference between the drag coefficient over the second and the first/third cylinders are decreased.

It is also interesting to observe how the third cylinder is affected by the flow comparing to the first cylinder. According to the graphs in Figure 7, the drag force over the cylinder in the middle position is always between the two other cylinders. However, the difference between the drag coefficients for the first and second cylinder is influenced by the separation distance, Bingham number, and the Reynolds number. As the Bingham number is increased, this difference is decreased. In contrast, by increasing the Reynolds number, this difference is increased due to the existing dominant viscous forces. In fact, except in cases where separation distance $d=2.0 D$, for other separation distances, there are some cases that the drag force over the first and the third cylinder is almost equal. For example, for $d=3.0 D$, cases where $B i=100$ and $B i=150$, the drag force for the cases with $R e=5$ are equal. By increasing the separation distance, these cases can be found in lower Bigham numbers as well. For example, for $d=6.0 D$ and $B i=30$, the first and third cylinders encounter a similar drag force.

Further, it was found that the maximum difference between the drag forces of the first and third cylinder is about $38 \%$ where $d=3.0 D, R e=5$ and $B i=150$. In this case, the difference between the drag force of the second and first cylinder was maximum too, whereas approximately a $59 \%$ difference was observed between these two drag forces.

As aforementioned, and likewise the cases for single cylinder, the Reynolds number has influence on the size and shape of the shaded region. Regarding the plots in Figures 7-10, one can mention that an increased Reynolds number has more effect on decreasing the size of the unyielded region around the cylinder rather than the size of the similar region in between the cylinders.

Increasing the Bingham number has another effect on the size and shape of the unyielded region in between the objects. It is noteworthy that in all cases, the height of the unyielded region is developed by increasing the Bingham number. In addition, some small islands are observed above the cylinders or around the cylinders when separation distance is increased to $d \geq 3.0 \mathrm{D}$ and Bingham number above 50 .

\section{Conclusions}

The variation of drag coefficient over three fixed cylindrical particles for various configurations in tandem position was investigated. Simulations were performed for $10 \leq B i \leq 150,5 \leq R e \leq 40$ and separation distances $2.0 D \leq d \leq 6.0 D$. The results had good agreement with previous studies for a single particle. 
For a single particle, it was shown that due to the dominant viscous forces in the lower Reynolds number, the effect of the Bingham number is more pronounced. The unyielded region shape and size were investigated, and several patterns were found dependent on Reynolds number and the Bingham number. It was shown that the unyielded region gets smaller in the flow direction by increasing the Bingham number, while it remains almost constant at the vertical direction.

For multiple cylinders, it was found that the separation distance has a significant effect on the drag variation on the cylinders. Several conditions (in terms of Bingham number, separation distance and Reynolds number) were found in which the first and the third cylinder are affected with the same drag force. Concerning the drag force applied to the cylinder in the middle position, higher difference between the drag coefficients of this cylinder and the other two cylinders was found for the higher Reynolds number.

Author Contributions: Conceptualization, N.H. and L.-G.W.; Methodology, N.H.; Software, N.H.; Validation, N.H.; Formal Analysis, N.H.; Investigation, L.-G.W.; Resources, L.-G.W.; Data Curation, N.H.; Writing-Original Draft Preparation, N.H.; Writing-Review \& Editing, N.H. and L.-G.W.; Visualization, N.H.; Project Administration, L.-G.W.; Funding Acquisition, L.-G.W.

Funding: This research was funded by Swedish KEMPE Foundations, Grant Number SMK-1739.

Acknowledgments: This research was conducted using the resources of High Performance Computer Center North (HPC2N), Sweden.

Conflicts of Interest: The authors declare no conflict of interest.

\section{References}

1. Dzuc, N.Q.; Boger, D.V. Yield Stress Measurement for Concentrated Suspensions. J. Rheol. 1983, 27, 321-349.

2. Wang, W.; Meng, B.; De Kee, D.; Khismatullin, D. Numerical investigation of plate edge and slot size effects in low yield stress measurements with a slotted plate device. Rheol. Acta 2012, 51, 151-162. [CrossRef]

3. Borgia, A.; Spera, F.J. Error analysis for reducing noisy wide-gap concentric cylinder rheometric data for nonlinear fluids: Theory and applications. J. Rheol. 1990, 34, 117-136. [CrossRef]

4. Heirman, G.; Vandewalle, L.; Gemert, D.V. Integration approach of the Couette inverse problem of powder type self-compacting concrete in a wide-gap concentric cylinder rheometer. J. Non-Newtonian Fluids Mech. 2008, 150, 93-103. [CrossRef]

5. James, P.W.; Jones, T.E.R.; Hughes, J.P. The determination of apparent viscosity using a wide gap, double concentric cylinder. J. Non-Newton. Fluid Mech. 2004, 124, 33-41. [CrossRef]

6. Barnes, H.; Walters, K. The yield stress myth? Rheol. Acta 1985, 24, 323-326. [CrossRef]

7. Barnes, H.A. The yield stress-A review or ' $\pi \alpha \nu \tau \alpha \rho \varepsilon \iota^{\prime}-E v e r y t h i n g$ flows? J. Non-Newton. Fluid Mech. 1999, 81, 133-178. [CrossRef]

8. Bingham, E.C. An Investigation of the Laws of Plastic Flow; US Government Printing Office: Pueblo, CO, USA, 1917; Volume 13.

9. Herschel, W.; Bulkley, R. Konsistenzmessungen von Gummi-Benzollösungen. Colloid Polym. Sci. 1926, 39, 291-300. [CrossRef]

10. Papanastasiou, T.C. Flows of Materials with Yield. J. Rheol. 1987, 31, 385-404. [CrossRef]

11. KÉ, D.D.; Turcotte, G. Viscosity of biomaterials. Chem. Eng. Commun. 1980, 6, 273-282. [CrossRef]

12. Mitsouls, E.; Abdali, S. Flow simulation of Herschel-Bulkley fluids through extrusion dies. Can. J. Chem. Eng. 1993, 71, 147-160. [CrossRef]

13. Zhu, H.; De Kee, D. A numerical study for the cessation of Couette flow of non-Newtonian fluids with a yield stress. J. Non-Newton. Fluid Mech. 2007, 143, 64-70. [CrossRef]

14. Zhu, H.; Kim, Y.D.; De Kee, D. Non-Newtonian fluids with a yield stress. J. Non-Newton. Fluid Mech. 2005, 129, 177-181. [CrossRef]

15. Stokes, S.G.G. Mathematical and Physical Papers; Cambridge University Press: Cambridge, UK, 1901.

16. Johnson, T.; Patel, V. Flow past a sphere up to a Reynolds number of 300. J. Fluid Mech. 1999, 378, $19-70$. [CrossRef]

17. Kim, I.; Pearlstein, A.J. Stability of the flow past a sphere. J. Fluid Mech. 1990, 211, 73-93. [CrossRef] 
18. Lee, S. A numerical study of the unsteady wake behind a sphere in a uniform flow at moderate Reynolds numbers. Comp. Fluids 2000, 29, 639-667. [CrossRef]

19. Fornberg, B. Steady viscous flow past a sphere at high Reynolds numbers. J. Fluid Mech. 1988, 190, 471-489. [CrossRef]

20. Beris, A.; Tsamopoulos, J.; Armstrong, R.; Brown, R. Creeping motion of a sphere through a Bingham plastic. J. Fluid Mech. 1985, 158, 219-244. [CrossRef]

21. Adachi, K.; Yoshioka, N. On creeping flow of a visco-plastic fluid past a circular cylinder. Chem. Eng. Sci. 1973, 28, 215-226. [CrossRef]

22. Mitsoulis, E. On creeping drag flow of a viscoplastic fluid past a circular cylinder: wall effects. Chem. Eng. Sci. 2004, 59, 789-800. [CrossRef]

23. Vakil, A.; Green, S.I. Two-dimensional side-by-side circular cylinders at moderate Reynolds numbers. Comp. Fluids 2011, 51, 136-144. [CrossRef]

24. Jossic, L.; Magnin, A. Drag of an isolated cylinder and interactions between two cylinders in yield stress fluids. J. Non-Newton. Fluid Mech. 2009, 164, 9-16. [CrossRef]

25. Koblitz, A.R.; Lovett, S.; Nikiforakis, N. Viscoplastic squeeze flow between two identical infinite circular cylinders. Phys. Rev. Fluids 2018, 3, 023301. [CrossRef]

26. Ozgoren, M. Flow structures around an equilateral triangle arrangement of three spheres. Int. J. Multiph. Flow 2013, 53, 54-64. [CrossRef]

27. $\mathrm{Wu}, \mathrm{Y}$. Numerical simulation of flows past multiple cylinders using the hybrid local domain free discretization and immersed boundary method. Ocean Eng. 2017, 141, 477-492. [CrossRef]

28. Bird, R.B.; Hassage, O. Dynamic of Polymeric Fluids, 2nd ed.; John Wiley and Sons Publication: Hoboken, NJ, USA, 1987.

29. Weller, H.G.; Tabor, G.; Jasak, H.; Fureby, C. A tensorial approach to computational continuum mechanics using object-oriented techniques. Comp. Phys. 1998, 12, 620-631. [CrossRef]

30. Mossaz, S.; Jay, P.; Magnin, A. Criteria for the appearance of recirculating and non-stationary regimes behind a cylinder in a viscoplastic fluid. J. Non-Newton. Fluid Mech. 2010, 165, 1525-1535. [CrossRef]

31. Nirmalkar, N.; Chhabra, R. Momentum and heat transfer from a heated circular cylinder in Bingham plastic fluids. Int. J. Heat Mass Transfer 2014, 70, 564-577. [CrossRef]

32. Thakur, P.; Mittal, S.; Tiwari, N.; Chhabra, R. The motion of a rotating circular cylinder in a stream of Bingham plastic fluid. J. Non-Newton. Fluid Mech. 2016, 235, 29-46. [CrossRef]

33. Zisis, T.; Mitsoulis, E. Viscoplastic flow around a cylinder kept between parallel plates. J. Non-Newton. Fluid Mech. 2002, 105, 1-20. [CrossRef]

34. Chatzimina, M.; Georgiou, G.C.; Argyropaidas, I.; Mitsoulis, E.; Huilgol, R. Cessation of Couette and Poiseuille flows of a Bingham plastic and finite stopping times. J. Non-Newton. Fluid Mech. 2005, 129, 117-127. [CrossRef]

(C) 2019 by the authors. Licensee MDPI, Basel, Switzerland. This article is an open access article distributed under the terms and conditions of the Creative Commons Attribution (CC BY) license (http://creativecommons.org/licenses/by/4.0/). 